\title{
P21 The Association of Vitamin K Antagonists with Aortic Arch Calcification in Acute Coronary Syndrome Patients
}

Konstantia-Paraskevi Gkini*, Dimitrios Terentes-Printzios, Charalambos Vlachopoulos, Aggeliki Rigatou, Vasiliki Gardikioti, Evangelia Sigala, Dimitrios Tousoulis

Hippokration Hospital, First Department of Cardiology, National and Kapodistrian University of Athens, Greece

\begin{abstract}
Purpose/Background/Objectives: Vitamin K antagonists (VKA) aggravate vascular calcifications burden, especially in haemodialysis patients potentially compounding their cardiovascular risk. We sought to investigate the relationship between VKA intake and aortic arch calcification (AAC) in patients with acute coronary syndrome (ACS).

Methods: Two hundred patients admitted with ACS were included in the study (mean age $66 \pm 15$ years). The extent of aortic arch calcification (AAC) on a postero-anterior plain chest X-ray was divided into four grades (0 to 3). Grades 0 to 1 and grades 2 to 3 were categorized as lower and higher AAC grade respectively. Anticoagulants were categorized to VKA and to non-VKA anticoagulants including new oral anticoagulants and low-molecular weight heparin.

Results: Twenty-eight (14\%) patients at admission were on anticoagulants. Seventeen patients (8.5\%) were treated with VKA and 12 patients $(6 \%)$ with non-VKA anticoagulants. Higher ACC grade was observed in patients treated with VKA after adjustment for age, gender and chronic kidney disease. (Odds ratio $[\mathrm{OR}]=3.64,95 \%$ Confidence intervals $[\mathrm{CI}]: 1.08$ to 12.20 , $p=0.037)$. Interestingly, there was a non-statistically significant trend for reduced risk of higher AAC grade in patients treated with non-VKA anticoagulants ( $\mathrm{OR}=0.24,95 \% \mathrm{CI}: 0.04$ to $1.47, p=0.12$ ). Age and history of chronic kidney disease were the most potent predictors of higher AAC grade (OR $=1.07,95 \%$ CI: 1.03 to $1.10, p<0.001$ and $\mathrm{OR}=6.07,95 \%$ CI: 2.35 to 15.67 , $p<0.001$, respectively).
\end{abstract}

Conclusion: Our study shows intake of VKA is associated with higher risk of AAC in ACS patients, while non-VKA anticoagulants might be associated lower AAC grade.

(c) 2019 Association for Research into Arterial Structure and Physiology. Publishing services by Atlantis Press International B.V. This is an open access article distributed under the CC BY-NC 4.0 license (http://creativecommons.org/licenses/by-nc/4.0/) 